\title{
¿Cuándo pensar en pseudoartrosis congénita de clavícula en pediatría? Presentación de 2 casos
}

\author{
When to think about pediatric congenital pseudoarthrosis of the clavicle? \\ Presentation of 2 cases
}

\author{
Dr. Pablo R. Justich Zabala ${ }^{a}$, Dr. Rodolfo P. Giniger Vidala , Dra. María J. Rubio Péreza ${ }^{a}$ Dr. José Guirao Péreza \\ y Dr. César Salcedo Canovas ${ }^{a, b}$
}

\begin{abstract}
RESUMEN
La pseudoartrosis congénita dela clavícula es una malformación rara y benigna, caracterizada por la ausencia del tercio medio de la clavícula. Suele ser unilateral y mayoritaria en el lado derecho. La etiología es desconocida y se postulan diversas teorías etiopatogénicas (vascular, embriológica y genética). Puede detectarse en el período neonatal o, más frecuentemente, durante la infancia. En ocasiones, puede ser sintomática. Puede requerir tratamiento mediante reconstrucción quirúrgica por injerto óseo.

Se presentan 2 casos, uno de diagnóstico neonatal y otro de 3 años de edad realizados con $24 \mathrm{~h}$ de diferencia. Se destaca la consideración de este diagnóstico como diferencial de fractura obstétrica o postraumática, displasia cleidocraneal y neurofibromatosis de tipo 1 .

Palabras clave: pseudoartrosis, clavícula, anomalías congénitas.
\end{abstract}

\begin{abstract}
The congenital pseudoarthrosis of the clavicle is a rare and benign malformation, characterized by the absence of the middle third of the clavicle. It is usually unilateral and the majority on the right side. The etiology is unknown, postulating diverse etiopathogenic theories (vascular, embryological and genetic). It can be detected in the neonatal period or, more frequently, during childhood. Occasionally it can be symptomatic. It may require treatment by surgical reconstruction by bone graft.

Two cases are presented, one of neonatal diagnosis and another one of 3 years of age performed with 24 hours of difference. We emphasize on its consideration as a differential diagnosis of obstetric or post-traumatic fracture, cleidocranial dysplasia and neurofibromatosis type I.
\end{abstract}

Key words: pseudoarthrosis, clavicle, congenital abnormalities.

http: / / dx.doi.org/10.5546/ aap.2020.e194

Cómo citar: Justich Zabala PR, Giniger Vidal RP, Rubio Pérez MJ, Guirao Pérez J, Salcedo Canovas C. ¿Cuándo pensar en pseudoartrosis congénita de clavícula en pediatría? Presentación de 2 casos. Arch Argent Pediatr 2020;118(2):e194-e198.

a. Hospital La Vega, Murcia, España.

b. Hospital Clínico Universitario "Virgen de la Arrixaca", Murcia, España.

Correspondencia:

Dr. Pablo R. Justich Zabala: pablojustich@gmail.com

Financiamiento: Ninguno.

Conflicto de intereses: Ninguno que declarar.

Recibido: 19-4-2019

Aceptado: 11-9-2019

\section{INTRODUCCIÓN}

La pseudoartrosis congénita de la clavícula (PCC) es una malformación rara y benigna, caracterizada por una tumoración, habitualmente, indolora, formada por dos segmentos de clavícula independientes entre sí. ${ }^{1}$ Fue reconocida por primera vez como entidad diferenciada por Fitzwilliams ${ }^{2}$ en 1910 y luego descrita con mayor detalle por Alldred ${ }^{3}$ en 1963, que la diferenció, entre otras entidades, de la fractura de clavícula obstétrica.

En la actualidad, hay publicados unos 200 casos. Suele ser unilateral y mayoritaria en el lado derecho, y son excepcionales los casos bilaterales. ${ }^{4}$ La etiología es desconocida y se postulan diversas teorías etiopatogénicas, con mayor o menor apoyo. Una teoría "vascular", que hace referencia a la presión ejercida por la arteria subclavia sobre la clavícula durante el desarrollo. ${ }^{5}$ Una teoría "embriológica", que postula un posible defecto en la coalescencia de dos supuestos centros de osificación o la inadecuada reabsorción de un único centro. ${ }^{6}$ Finalmente, una teoría "genética", que se sustenta en la aparición de casos familiares. ${ }^{7}$

Puede detectarse en el período neonatal o, más frecuentemente, durante la infancia cuando, al perderse el tejido adiposo en la zona, se advierte la prominencia en el tercio medio de la clavícula, no dolorosa y que no se acompaña de impotencia funcional. Muy ocasionalmente, pueden aparecer molestias, acentuadas con el ejercicio, y una asimetría del hombro con el descenso del lado afectado. ${ }^{8} \mathrm{La}$ anomalía se produce en la clavícula derecha en un $80 \%$ de los casos.

Se aportan, a continuación, dos nuevos casos de PCC con edad y forma de presentación diferentes y con un intervalo de 24 horas entre ambos diagnósticos. Se destaca la sospecha clínica y, sobre todo, la importancia de la discusión, en el pase de sala y entre las especialidades participantes, de aquellos casos con características peculiares. 


\section{Caso 1}

Recién nacida de término con peso adecuado para la edad gestacional. Nacida a las 39 semanas y 2 días de gestación por cesárea electiva sin trabajo de parto, indicada por crecimiento intrauterino retardado. Peso al nacer: $2520 \mathrm{~g}$. Presentación cefálica, Apgar 9/10. Embarazo controlado, sin particularidades.

En la exploración inicial, se constató una solución de continuidad en la clavícula derecha con palpación de cabos móviles, sin signos de contractura muscular ni crepitación. La exploración y la falta de factores de riesgo o antecedentes obstétricos compatibles con fractura de clavícula hicieron sospechar una displasia clavicular. No se observaron malformaciones craneofaciales ni alteración en la clavícula izquierda.

Fue evaluada por Cardiología, que diagnosticó un soplo funcional. La radiografía de tórax (Figura 1) mostró una imagen compatible con PCC. El Servicio de Diagnóstico por Imágenes lo confirmó. La paciente ha sido seguida por Traumatología Infantil, sin intervenciones hasta el momento. La segunda imagen (Figura 2), tomada a los 8 meses de vida, muestra la persistencia del defecto con una diástasis menor que la inicial.

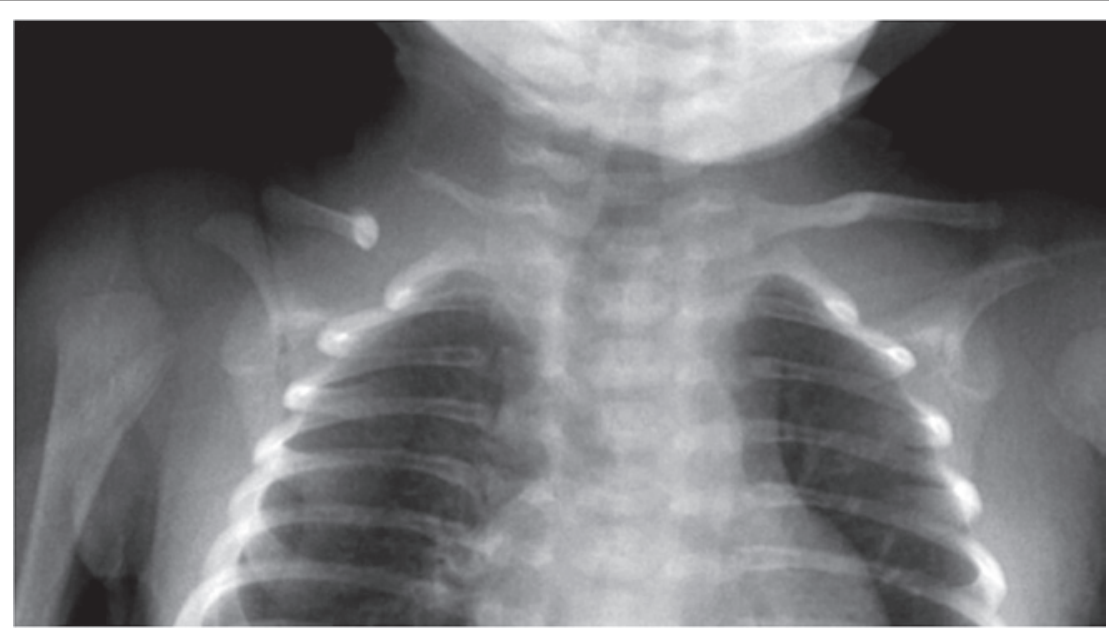

Radiografía del tórax de frente, a los 3 días de vida. Muestra, en la clavícula derecha, el final engrosado del extremo medial del segmento distal y la separación entre ambos segmentos.

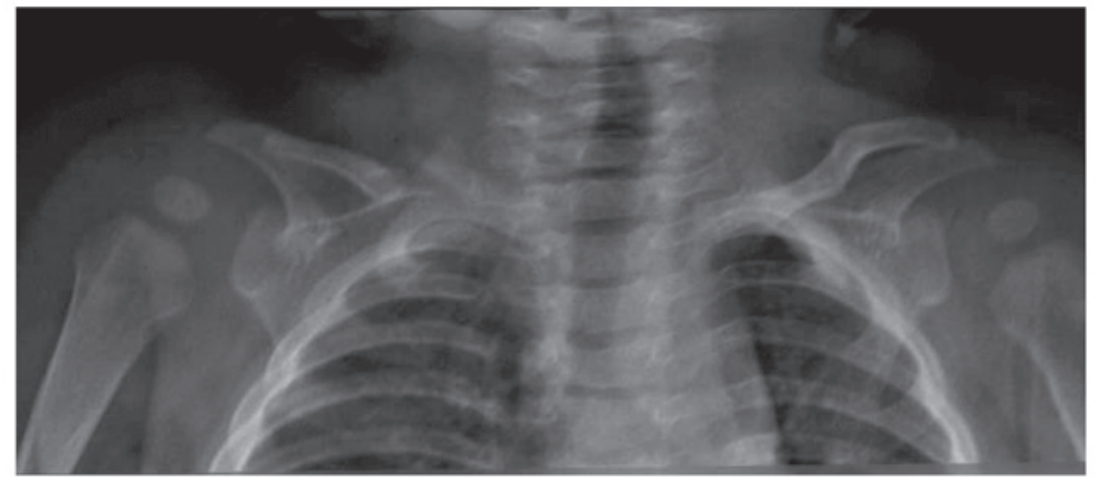

Radiografía de la clavícula realizada a los 8 meses de edad. Muestra una mayor alineación entre ambos segmentos de la clavícula derecha y que se mantiene la separación entre ambos. 


\section{Caso 2}

Paciente de 3 años de edad, que acudió a Urgencias por una patología respiratoria y en cuya radiografía de tórax se observaba una imagen de solución de continuidad en la clavícula derecha (Figura 3). No presentaba dolor ni impotencia funcional. Se consideró, en aquel momento, como fractura de clavícula y se indicó la inmovilización. Dada la cercanía del caso 1, no habiendo antecedente de traumatismo y con una imagen atípica, se realizó una interconsulta con el Servicio de Diagnóstico por Imágenes, que diagnosticó una PCC.
Como antecedentes perinatales, se trataba de una niña nacida a término (39 semanas de gestación) y peso adecuado para la edad gestacional (3410 g). Embarazo controlado y sin complicaciones. Parto eutócico, con presentación cefálica, sin complicaciones. Apgar 9/10. Sin antecedentes patológicos de relevancia durante los primeros años de vida.

Se recuperó una radiografía de tórax de 1 año antes del evento actual que ya mostraba la mencionada malformación clavicular (Figura 4). Actualmente, se encuentra en seguimiento por Traumatología Infantil.

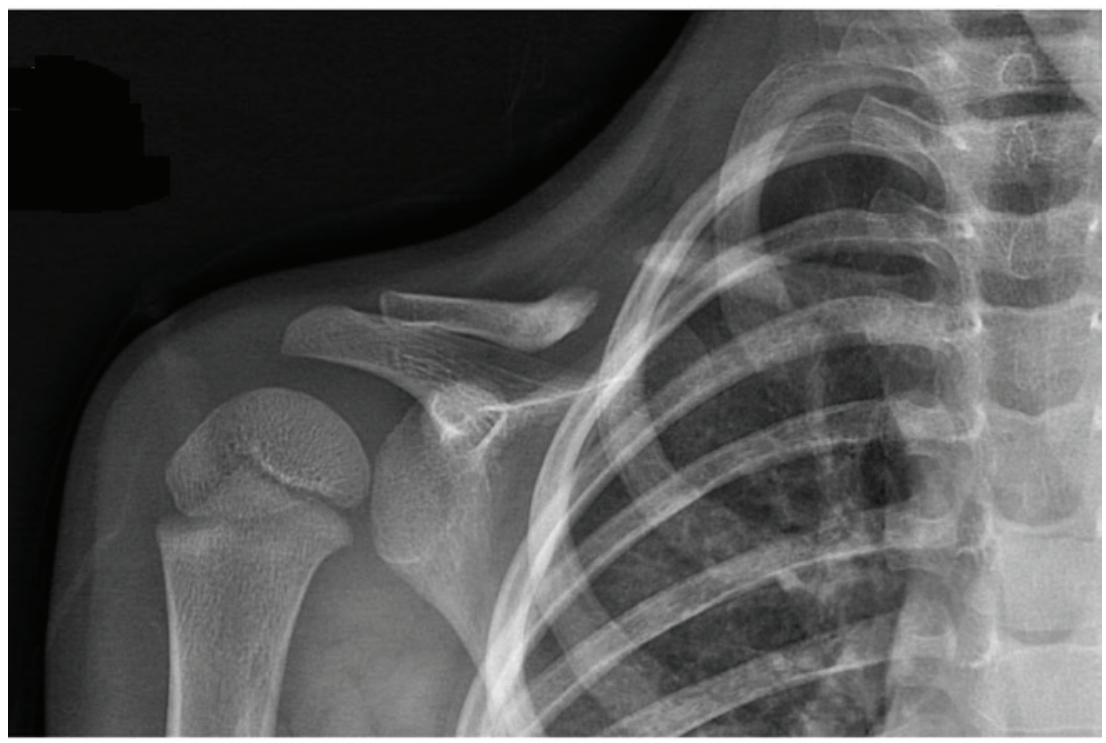

Radiografía de la clavícula derecha, realizada a los 3 años de edad. Se observa la separación de ambos segmentos con un leve engrosamiento del extremo medial del segmento distal.

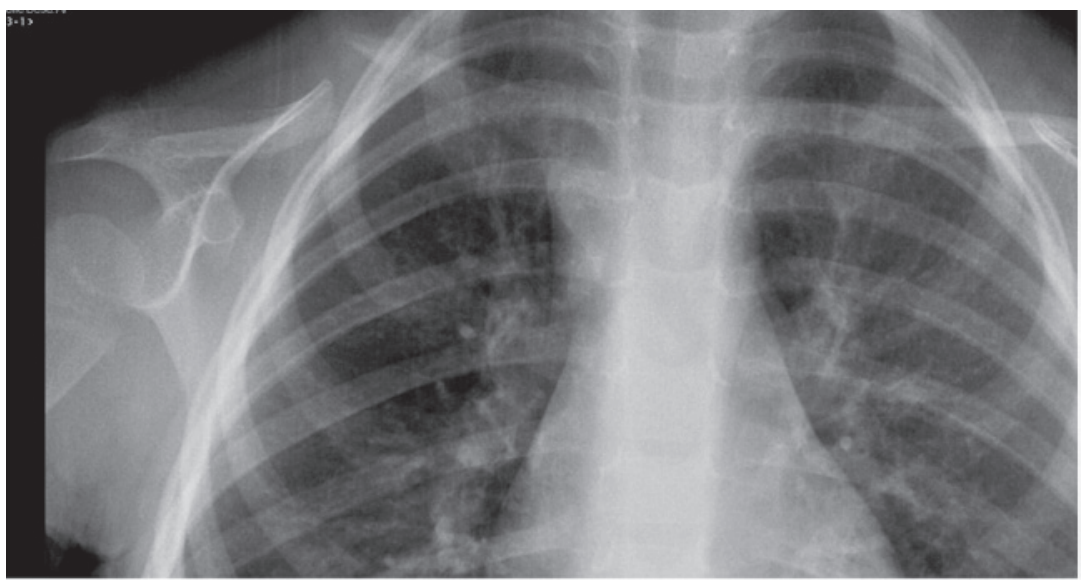

Radiografía del tórax de frente realizada por otras causas a los 2 años de edad, en la que se observaba la separación entre ambos segmentos en la clavícula derecha. 


\section{DISCUSIÓN}

Si bien se trata de un defecto congénito, esta patología puede pasar inadvertida o, en el período neonatal, ser confundida con las fracturas obstétricas. Suele ponerse en evidencia por cuestiones más estéticas que funcionales.

La sospecha diagnóstica de PCC debe realizarse ante la localización (generalmente, derecha) de una tumoración no dolorosa y con escasa repercusión funcional, que se suele observar desde las primeras semanas de vida. Con el crecimiento del paciente, esta tumoración suele aumentar de tamaño. El examen radiológico muestra la ausencia de la formación de callo y una solución de continuidad en la clavícula con extremos óseos ensanchados y enfrentados.

El pediatra deberá diferenciar esta patología de la fractura de clavícula durante el período neonatal, dada su frecuencia y similitud. ${ }^{9}$ También se deben incluir, entre los diagnósticos diferenciales, la displasia cleidocraneal, la neurofibromatosis de tipo 1 (NF1) y la pseudoartrosis postraumática. ${ }^{10}$ Tanto en la fractura obstétrica como en las postraumáticas, hay dolor y repercusión funcional, además de observarse una hipertrofia de tejido osteofibroso. Las otras causas suelen ser indoloras. En cuanto a las NF1, existen lesiones cutáneas típicas entre otros signos clínicos. La displasia cleidocraneal, a su vez, presenta otras malformaciones craneofaciales.

El segundo caso presentado ilustra dos hechos que se desean destacar en el presente artículo. Por un lado, la importancia de la observación sistemática de la radiografía y no solo centrada en la patología sospechada. En nuestro caso, permitió encontrar hallazgos patológicos que no estaban ligados al motivo de consulta. Por otro, esta observación se vio favorecida por el comentario en el pase de sala del caso 1 poco tiempo antes. Los pacientes, como este último, que se presentan fuera del período neonatal requieren, además de un alto índice de sospecha y de la observación detenida de las imágenes, la realización de una detallada historia clínica y, sobre todo, perinatal.

Históricamente, ambos abordajes (reparación quirúrgica u observación sin intervención en los asintomáticos) fueron recomendados por diferentes autores. La observación en los pacientes asintomáticos y sin requerimientos estéticos parece ser una opción válida considerando que la cirugía no está exenta de complicaciones ni de rastros estéticos. La cirugía (escisión de la pseudoartrosis, fijación interna e injerto de hueso) se reserva a pocos casos, en su mayoría, por cuestiones estéticas o para corregir aquellas deformidades del hombro que interfieren con la funcionalidad. ${ }^{11}$ La intervención se aconseja a partir de los 4 años de edad ${ }^{12}$ y la técnica quirúrgica que ofrece mejores resultados, en la actualidad, consiste en la resección de los bordes pseudoartrósicos, osteosíntesis y aporte biológico mediante el injerto de la cresta ilíaca autóloga.

Algunos autores abogan por un tratamiento quirúrgico precoz en todos los casos para evitar, así, las complicaciones vasculares (síndrome del desfiladero torácico, tromboembolismo, etc.) descritas en algunos pacientes ya en la adolescencia. ${ }^{13,14}$ Por el contrario, otros especialistas recomiendan un tratamiento conservador mientras el paciente esté asintomático, dado que también han sido descritas, especialmente en el pasado, complicaciones de la cirugía; la más grave de ellas, la neuropraxia aguda del plexo braquial. ${ }^{15}$

Plantear la PCC como uno de los diagnósticos diferenciales en la patología clavicular puede colaborar con la adecuada información y orientación de los padres. La clínica y la radiología deberían ser suficientes para llegar al diagnóstico. El conocimiento de esta patología podría evitar exámenes complementarios y/o tratamientos no siempre necesarios.

La observación sistemática de la radiografía, no solo centrada en la patología sospechada, permite obtener hallazgos patológicos no necesariamente relacionados con la prescripción del estudio. La discusión con los miembros del servicio y con las otras disciplinas relacionadas contribuye a ampliar la detección temprana de este tipo de patologías.

\section{Agradecimientos}

A los Dres. Nelson Pinedo, Francisco Pena y Carina Cerchi, que han participado en la atención de estos pacientes.

\section{REFERENCIAS}

1. Fernández Moral V, Braña Vigil A, Castañon Muñiz ML, Braña Vigil A. Pseudoartrosis congénita de clavícula. A propósito de un caso. Rev Esp Cir Osteoart. 1993; 28(163):51-3.

2. Fitzwilliams DCL. Hereditary cranio-cleido-dysostosis. Lancet. 1910; 176(4551):1466-75.

3. Alldred AJ. Congenital pseudoarthrosis of the clavicle. J Bone Joint Surg Br. 1963; 45-B:312-9.

4. Nieto Gil A, Gómez Navalón A, Zorrilla Ribot P. Seudoartrosis congénita de clavícula bilateral. Caso clínico. Rev Esp Cir Ortop y Traumat. 2016; 60(6):397-9. 
5. Vergara Amador E, Galván Villamarín F, Piña Quintero M. Seudoartrosis congénita de clavícula. Patología de alta confusión diagnóstica. Rev Fac Med. 2006; 54(3):206-10.

6. Sung T,ManE, Chan A, LeeWK. Congenital pseudoarthrosis of the clavicle: a rare and challenging diagnosis. Hong Kong Med J. 2013; 19(3):265-7.

7. Fernández-Cuesta Valcarce MA, Pascual Pérez JM, López Gabriel ML, De Hoyos López C. Seudoatrosis congénita de clavícula en 2 gemelos. An Pediatr (Barc). 2003; 58(1):78-9.

8. Cadilhac C, Fenoll B, Peretti A, Padovani JP, et al. Pseudarthrose congénitale de la clavicule. Rev Chir Orthop Reparatrice Appar Mot. 2000; 86(6):575-80.

9. Chalfant JS, Sanchez TR. Congenital clavicular pseudoarthrosis-How to differentiate it from the more common clavicular fractures. Pediatr Emerg Care. 2019; 35(2):e37-9.
10. Figueiredo MJPSS, Braga SR, Akkari M, Prado JCL, et al. Pseudoartrose congênita de clavícula. Rev Bras Ortop. 2012; 47(1):21-6.

11. Currarino G, Herring J. Congenital pseudarthrosis of the clavicle. Pediatr Radiol. 2009; 39(12):1343-9.

12. Studer K, Baker M, Krieg A. Operative treatment of the congenital pseudarthrosis of the clavicle: a single-centre experience. J Pediatr Orthop B. 2017; 26(3):245-9.

13. Di Gennaro GL, Cravino M, Martinelli A, Berardi E, et al. Congenital pseudarthrosis of the clavicle: a report on 27 cases. J Shoulder Elbow Surg. 2017; 26(3):e65-70.

14. WatsonHI,HopperGP,KovacsP.Congenitalpseudarthrosis of the clavicle causing thoracic outlet syndrome. BMJ Case Rep. 2013; 2013:bcr2013010437.

15. Ullot Font R, Cepero Campà S, Gargantilla Vázquez A, Hernández Bou S. Seudoartrosis congénita de clavícula. An Pediatr (Barc). 2006; 64(3):273-6. 